\title{
Colour Contrast Enhancement Method by Scaling the DC Coefficients in CIE-LAB Colour Space
}

\author{
Anzar Alam \\ Department of Electronics \& \\ Communication \\ SISTEC Bhopal India
}

\author{
Mohammad. Abdullah \\ Department of Electronics \& \\ Communication \\ SISTEC Bhopal India
}

\author{
Ravi Shankar Mishra, \\ Ph.D \\ Department of Electronics \& \\ Communication \\ SISTEC Bhopal India
}

\begin{abstract}
Colour and brightness of the captured images from low cost digital cameras are usually different from the colour and brightness of the original scene. Many factors are responsible for the low contrast images such as non uniform illumination, large or small exposure of some specific regions, and the unwanted movements of camera users. Due to these reasons the captured image to looks annoying to human visual system. Thus, contrast enhancement methods are required for improving the visual appearance of the colour images. In this paper the performance of contrast limited adaptive histogram equalization (CLAHE) method is compared with method of DC coefficient scaling in the compressed DCT domain for enhancing the contrast of colour images. In addition, as a modification DC coefficient scaling is also implemented in the CIE-Lab colour space. The contrast is only enhanced in $\mathrm{L}$ component and keeping the colour information preserved. This improves the entropy of standard method. For comparing the performance along with SNR and entropy the absolute standard deviation difference is also used as parameters. The methods are tested on various true colour images from different environment.
\end{abstract}

\section{General Terms}

Image processing, Image enhancement, Colour spaces

\section{Keywords}

Contrast enhancement, Lab colour spaces, Contrast limited Adaptive Histogram Equalization, Discrete Cosine Transform, Entropy.

\section{INTRODUCTION}

The colour contrast enhancement techniques provide a way to improving the detection of object in the images and their identification in the low illumination conditions. Researchers have designed many methods for improving the image quality [1, 3, and 4]. The most common method of enhancing the image quality is to enhance its contrast [1]. The contrast is basically defined as the perceived difference between the image colours which are having close proximity to each other. Efficient contrast enhancement not stage differentiates high level design from each others. It also forces the image content to look pleasant to every viewer.

The methods of colour image enhancement are mainly classified in two groups as spatial domain methods and transform domain methods. The contrast limited adaptive histogram equalization (CLAHE) [5] method is the most popular spatial domain enhancement method. The methods based on DWT [2] and DCT [1] are most commonly used transform domain methods. DCT based methods are very common for enhancing the colour images. One of the common methods in compressed DCT domain is scaling the DC coefficient in DCT domain [1]. This paper have compared the standard DC coefficient scaling method in the $\mathrm{Y}-\mathrm{Cb}-\mathrm{Cr}$ colour space and DC coefficient scaling in CIE-Lab colour space for true colour images. CIE Lab is also known as the colour-opponent space because it imitates the way in which human visual system reads colour. Therefore this colour space permits more perceptual uniformities in comparison to other colour spaces. This allows for more accurate contrast adjustments and balanced colour corrections. Therefore using the CIE Lab colour space improves the information contain and the entropy of the enhanced image.

Rest of the paper is organized as follows: Section II have reviewed the various contrast enhancement methods and also describes the various types of colour spaces. In section III describes the spatial domain contrast enhancement techniques. In section IV a compressed domain method using DC coefficient scaling is described in detail. Section V details result of all these methods in comparative form are presented. Section VI gives conclusion and future work.

\section{LITERATURE REVIEW}

Various methods are proposed in literature for enhancing the images such as methods using the image histogram, local region based enhancement, and transform domain methods. in this section paper reviews some of the relevant methods of enhancing the true colour images..

\section{A. Review of Contrast enhancement methods}

Trahanias et al. [6] proposed a 3D histogram equalization method in RGB cube. Thomas et al. [7] proposed an enhancement method by considering the correlation between the luminance and saturation components of the image locally. Contrast limited adaptive histogram equalization (CLAHE) method by K. Zuiderveld [5] and Pisano et al. [11] were proposed to improve the image contrast for medical image applications and to overcome the amplification of noise. 
Table 1 Comparison of existing methods

\begin{tabular}{|c|c|c|c|}
\hline Author/ reference & $\begin{array}{c}\text { Method } \\
\text { Used }\end{array}$ & $\begin{array}{c}\text { Kind of images } \\
\text { application areas) }\end{array}$ & Used colour spaces \\
\hline Jayanta M.[1] & $\begin{array}{c}\text { Compressed } \\
\text { DCT }\end{array}$ & $\begin{array}{c}\text { True colour images with } \\
\text { low contrast }\end{array}$ & $\begin{array}{c}\text { Scaling the DC coefficient in Y-Cb- } \\
\text { Cr Space }\end{array}$ \\
\hline Prateek et al [2] & $\begin{array}{c}\text { Compressed } \\
\text { DWT }\end{array}$ & $\begin{array}{c}\text { True colour } \\
\text { Images }\end{array}$ & $\begin{array}{c}\text { Scaling the DWT coefficient in Y- } \\
\text { Cb-Cr Space }\end{array}$ \\
\hline Hatim et al. [5] & Spatial Domain & Low contrast & $\begin{array}{c}\text { Using Fusion of CLAHE in RGB and } \\
\text { HSV colour space }\end{array}$ \\
\hline $\begin{array}{c}\text { Our proposed } \\
\text { work }\end{array}$ & $\begin{array}{c}\text { Compressed } \\
\text { DCT }\end{array}$ & $\begin{array}{c}\text { Different colour images } \\
\text { with low contrast }\end{array}$ & $\begin{array}{c}\text { Scaling DC coefficient in LAB space } \\
\text { keeping the colour unchanged }\end{array}$ \\
\hline
\end{tabular}

Hitam et al. [5] have presented a combination of CHAHE in RGB and HSV colour spaces for underwater images. They have used image fusion technique to produce the enhanced image by fusing images in RGB and HSV colour spaces. The above mentioned methods are useful for colour image enhancement. However, colour shifting and brightness change is major problem. Colour shifting means, that one colour is changed to another. Irene et al have used CIE-Lab colour space for the detection of the optical disc in retinal images

However, in the last decade images are increasingly available in the compressed format. Therefore, various enhancement algorithms are developed in the compressed domain $[1,12$, and 13]. Among these method of scaling the DC coefficient in the block DCT domain Jayanta et al. [1], has recently being popularly used. In this paper SNR and mean square error are used as parameter to compare three enhancement techniques. The comparison of reviewed methods of colour image enhancements is presented in the Table 1. It is clear that proposed method of image enhancement replaces $\mathrm{Y}-\mathrm{Cb}-\mathrm{Cr}$ with CIE-Lab colour space.

\section{B. Review of Colour Spaces}

For improving the visual representation of colour images many colour models have been designed by the researchers. Colour space is a generalized term used for representing particular combination of a colour model plus a mapping functions associated to colour images. Colour model and colour spaces are complementary to each other. Colour image contain more precise information than the gray images, since large number of colours can be generated by mixing the primary colours pigments. Most commonly used colour spaces are RGB [15] and $\mathrm{Y}-\mathrm{Cb}-\mathrm{Cr}$ colour spaces [1]. CIE (Commission Internationale de l'eclairage), has developed a new colour space named as CIE-Lab colour space. Selection of the colour model depends on the kind of image application and its requirements.

\section{i) $R G B$ Colour space}

This is a tri colour space called as RGB (red, green and blue) colour space. The RGB colour model can be approximated as cube and red, green and blue can be considered as the $\mathrm{X}, \mathrm{Y}$ and $\mathrm{Z}$ axes as shown in Figure 1.

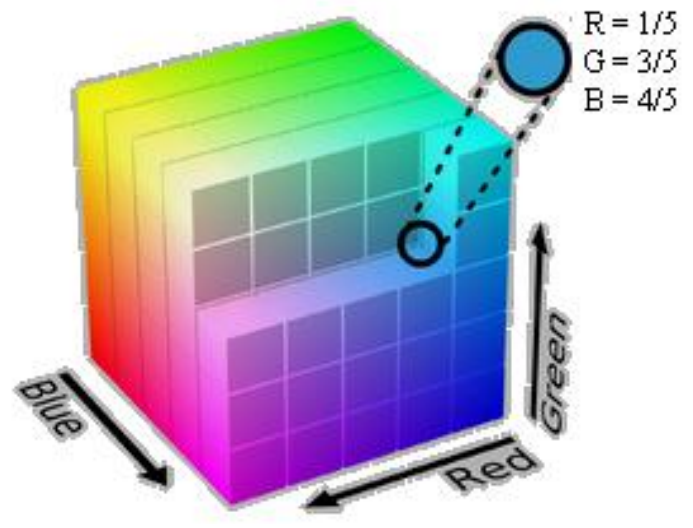

Figure 1 RGB Colour space as cubic model

RGB colour space is widely used for image compression. In RGB format every image contains three 2D images each corresponds to the Red, Green, and Blue colour spaces.

\section{ii). $\mathrm{Y}$ - $\mathrm{Cb}$-Cr Colour space}

The captured input colour image is converted into other format like $\mathrm{HSV}$ or $\mathrm{Y}-\mathrm{Cb}-\mathrm{Cr}$ formats for colour image enhancement. Because it is difficult to process the true RGB colour image directly and it is computationally complex too. Mostly Y-Cb-Cr. format is preferred over the HSV format due to its lesser complexity. In $\mathrm{Y}-\mathrm{Cb}-\mathrm{Cr}$ colour format, $\mathrm{Y}$ represents the actual brightness of the pixel thus called luminance signal, the $\mathrm{Cb}$ and $\mathrm{Cr}$ represent the chrominance signals where $\mathrm{b}$ and $\mathrm{r}$ represents the blue and red colour respectively. The $\mathrm{Y}-\mathrm{Cb}-\mathrm{Cr}$ colour space conversion allows greater compression without a significant effect on perceptual image quality. Therefore in most of the compressed domain methods the RGB images are converted to the $\mathrm{Y}-\mathrm{Cb}-\mathrm{Cr}$ formats.

\section{iii) CIE-Lab Colour space}

A CIE-Lab Colour space [16] is represented in the Figure 2. In the Figure the vertical L axis represents Lightness, between $0-100$. The horizontal axes represents $\mathrm{a}^{*}$ and $\mathrm{b}^{*}$ colour channels respectively. These colour channels are at orthogonal to each other and intersects each other at the centre, which is the neutral grey, black or white colour. They are based on the principal that a colour cannot be both red and green, or blue and yellow 


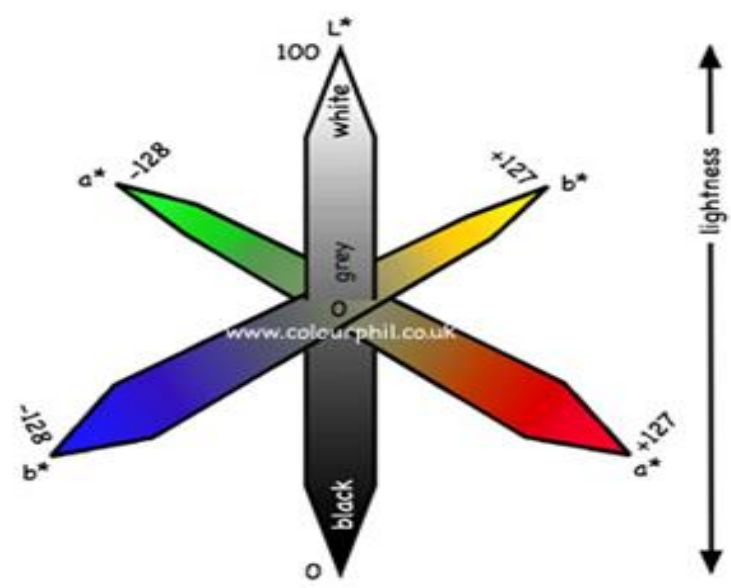

Figure 2 Representation of LAB colour space

The major advantage of the LAB colour space is that it is generating colours as similar to as human visual system. Therefore LAB colour spaces are extensively used in industries. Some of the application areas are; providing exact colour shades for paints for automotive and house hold industries, used in dyes in textiles industries. Also these colour spaces are widely used for object segmentation applications [3, 15].

\section{Spatial Domain Image Enhancement Techniques}

A spatial domain method directly transforms the intensity of the pixels for enhancing the images thus they are also called as direct methods. Histogram equalization (HE) is most frequently used spatial domain method for enhancing the given low contrast images [9, and 10]. Method flattens and stretches the dynamic range of intensity histogram and therefore results enhanced contrast. The resulting equalized images contain the even distribution of the gray levels. Although, there are still situations where basing image enhancement on a uniform histogram may not be the best approach. In these situations, histogram equalization may cause severe distortion HE [9] has been widely applied to enhance the images. Using histogram equalization can be a good approach when automatic enhancement is desired. However, HE may significantly change the image brightness and causes problems in applications where brightness preservation is necessary.

\section{a) Contrast Limited Adaptive Histogram Equalization (CLAHE)}

Another popular spatial domain method of contrast enhancement is CLAHE method [5, 15]. Method produces the optimal equalization in terms of maximum entropy and also limits the contrast of an image. The CLAHE method is very useful where the brightness requirement is high like as in geographical channels or underwater environments.

In this paper CLAHE method [5] is implemented for true colour images. Equalization is implemented individually for all three RGB colour spaces. These equalized RGB components are merged together to result the colour equalized image [15]. CLAHE was originally developed for successfully enhancing the low contrast medical images [5]. Method partitions the images into related regions and finds the equalization to each region. This flattens the distribution of grey levels and thus makes hidden features of the image more visible. In this paper images are equalized in RGB colour spaces individually. Basic algorithm for enhancing the contextual image regions by using the CLAHE technique is described below:

Step 1: Divide the each input image into a number of nonoverlapping contextual regions of equal size of the $8 \times 8$ blocks, each of which corresponds to the neighbourhood of 64 pixels.

Step 2: Individually calculate the intensity histogram of each contextual regions.

Step 3: Set the clip limits for clipping the histograms, (for example $\mathrm{c}=0.002$ ). The clip limit is a threshold parameter for effectively altering the contrast of the image. Higher clip limits increases the contrast of local image regions thus it must be set to minimum optimal value.

Step 4: Modified the each histogram by the selecting a suitable transformation functions.

Step 5: Each histogram is transformed in such a way that its height did not exceed the selected clip limit.

The mathematical expression for transformed gray levels for standard CLAHE method with Uniform Distribution can be given as

$$
g=\left[g_{\max }-g_{\min }\right] * P(f)+g_{\min }
$$

Where $g_{\max }=$ Maximum pixel value $g_{\min }=$ Minimum pixel value $\mathrm{g}$ is the computed pixel value $P(f)=$ CPD $\quad$ (Cumulative

probability distribution)

For exponential distribution gray level can be adapted as

$$
g=g_{\min }-\left(\frac{1}{\alpha}\right) * \ln [1-P(f)]
$$

Where $\alpha$ is the clip parameter? CLAHE method operates on small regions in the image, called "tiles", rather than the entire image. Each tile's contrast is enhanced, so that the histogram of the output region approximately matches the histogram specified by the distribution type. The Cumulative probability distribution of Rayleigh distribution is given as;

$$
y=P(f(x \backslash b))=\int_{0}^{x} \frac{x}{b^{2}} e^{\left(\frac{-x^{2}}{2 b^{2}}\right)}
$$

Step 6: The neighbouring tiles were combined using bilinear interpolation and the image gray scale values were altered according to the modified histograms.

An example of the CLAHE enhanced image is shown in the Figure 3 for lg-image16 with non uniform brightness. It is clear that the CLAHE method improves the information in the enhanced image by improving the contrast.
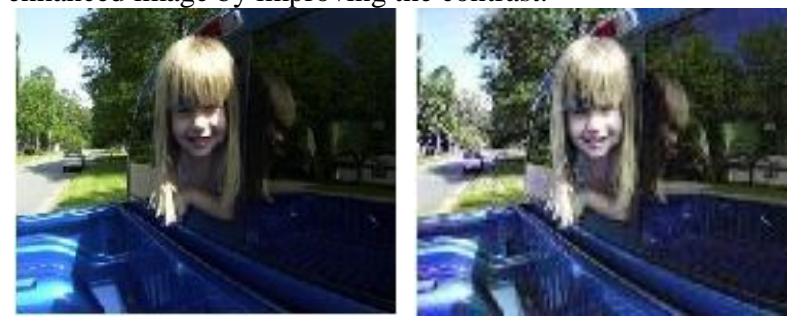

a) Original ig-image16, [1] b) CLAHE enhanced image 
Figure 3 Example of CLAHE Enhancement

\section{COMPRESED DOMAIN IMAGE ENHANCEMENT TECHNIQUES}

Researchers have designed various compressed domain contrast enhancement methods using the discrete cosine transform (DCT) [1, 12] and discrete wavelets transform (DWT) $[2,14]$. These methods demonstrated the improved visual appearance of the images. Different methods have been proposed in literatures, designed for both coloured and gray scale images. DCT based method transforms an image from $\mathrm{R}-\mathrm{G}-\mathrm{B}$ to $\mathrm{Y}-\mathrm{Cb}-\mathrm{Cr}$ color space. This represents the colour in terms of Luminance and Chrominance components thus provide a better platform for enhancing the color images. In this paper it is proposed to enhance true color images in the compressed DCT domain using scaling of DC coefficients. The performance of the method is compared with existing spatial domain methods.

Method finds the DCT coefficients of the Y components using 8 X8 blocks. Then the DC coefficient of each DCT block is used for adjusting the local background illumination. Since the DC value gives the mean of the brightness distribution of the block. This adjustment may be performed by mapping the brightness values to the value in the desired range. This mapping function should be monotonic in the given range. Let us find the maximum brightness value of the input image $I$ as reference.

$$
\max (I)=I_{\max }
$$

The mapping function which must be capable of keeping the variations of the DC coefficients smooth or continuous are used to map the DC coefficient is done using. Paper uses Twicing function [1] as the mapping function.

$$
D C=\tau(x) * I_{\max }
$$

Where mathematically Twicing function $\tau(x)$ can be defined as;

$$
\tau(x)=x *(2-x)
$$

Find the DC coefficients and then scale the only DC coefficients of the DCT coefficients as;

$$
\mathrm{K}=\left(\mathrm{f}\left(\frac{\mathrm{Y}(0,0)}{\mathrm{N} I_{\max }}\right)\right) /\left(\frac{\mathrm{Y}(0,0)}{\mathrm{N} I_{\max }}\right)
$$

Basic concept of contrast enhancement by scaling the DC coefficient in DCT domain algorithm [12], is to filter the image by scaling the DCT coefficients according to the defined contrast measure. Paper adjusts the DC coefficient of the ' $\mathrm{Y}$ ' component of the image. This is done because the DC coefficient $(\mathrm{Y}(0,0))$ contains majority of the information. This technique also works faster than the spatial domain techniques as computing DCT coefficients is a faster process than working in the spatial domain.

\section{PROPOSED METHOD}

In order to improve the performance of the described standard DC coefficient scaling method, this paper proposed to increase the image contrast in $\mathrm{CIE}=\mathrm{Lab}$ color space instead of $\mathrm{Y}-\mathrm{Cb}-\mathrm{Cr}$. The block diagram of the proposed method is given in Figure 4. Using CIE-Lab space before image enhancement method increases the aesthetic appurtenance of the images. This allows enhancing the color feature of the structural components across the different spectral channels of the image. The DC coefficients are enhanced in lightness component $\mathrm{L}$ only and the color information in $\mathrm{a}^{*}$ and $\mathrm{b}^{*}$ channels are kept unchanged for preserving the color information. Finally the Lab color spaces are converted back to RGB images

\section{RESILTS AND DISCUSSION}

Paper presents a comparison of experimental results for different methods of enhancing the various true colour images. The image database used in this paper is shown in Figure 5 Figure 6 compares the simulation results of the enhanced images for CLAHE and the DC coefficient scaling methods for Y-Cb-Cr- and $\mathrm{LAB}$ formats for Face image.. Although CHALE method performs better in terms of entropy, but it suffers from colour shifting. The DCT based methods are free from colour shifting and performs better. The comparison of histograms for these methods is given in

the Figure

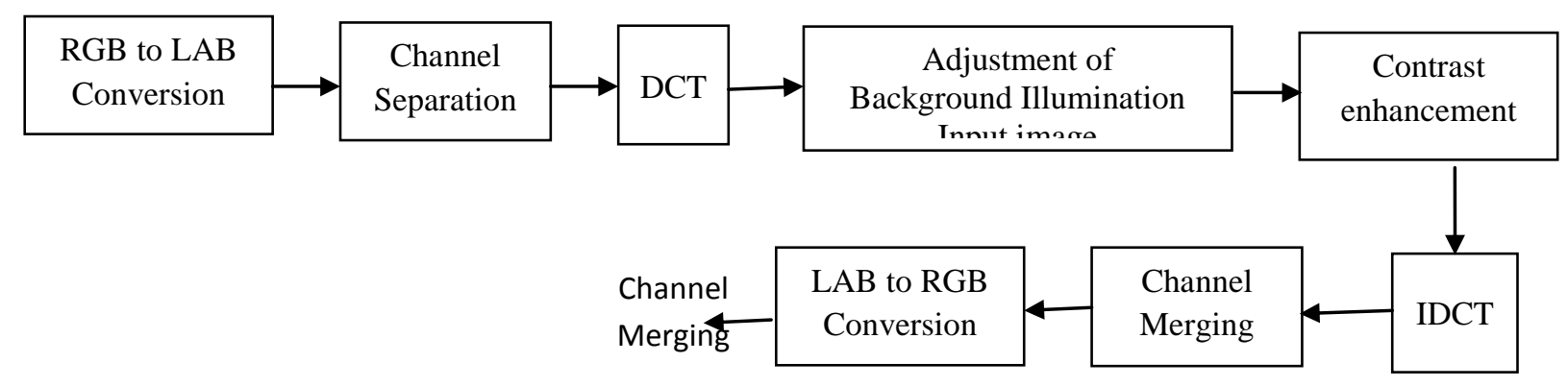

Figure 4 Block Diagram of the proposed enhancement method

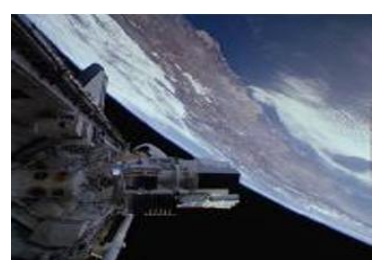

a) Satellite image

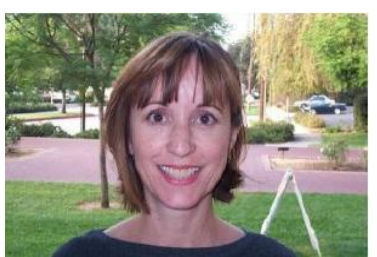

b) Faceimage1

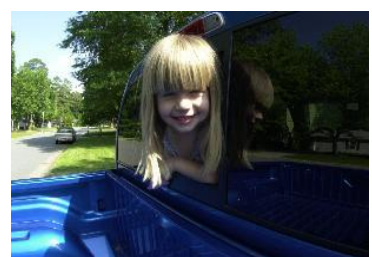

c) Lg-image 16

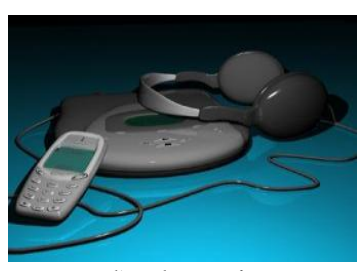

d) Player image

Figure 5 Original input images used for comparison 
The effect of colour shifting for the CLAHE enhanced image can be clearly observed from Figure 6 (b) as color of the face and trees. The LAB color space improves the visual apperancce

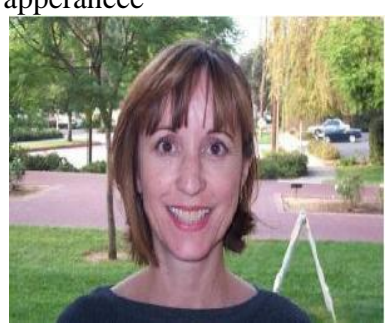

a) Original image

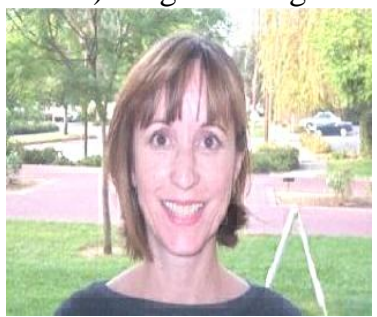

c) DCT enhanced with $\mathrm{Y}-\mathrm{Cb}-\mathrm{Cr}$ d) enhanced with $\mathrm{Lab}$ Figure 6 Results of enhancement methods for Face image.I

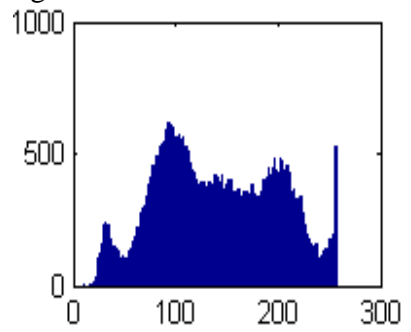

a) Original histogram
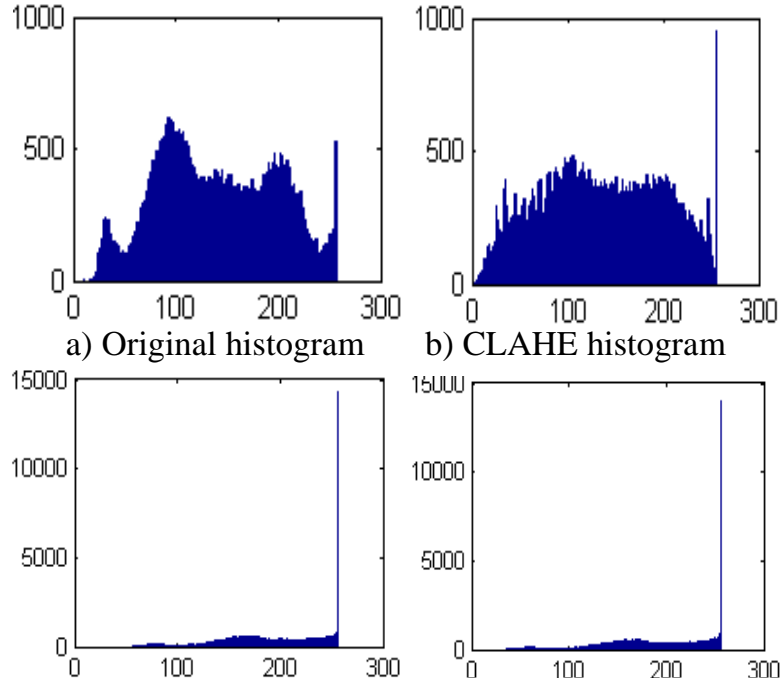

c) Histogram with DCT Y-Cb-Cr d) Enhanced with Lab

Figure 7 Comparison of enhanced histograms for Face image

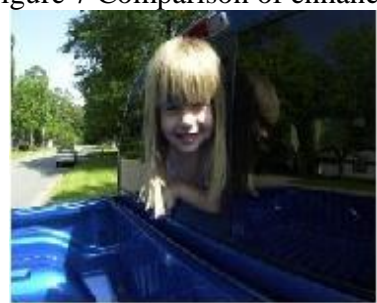

a) Original image

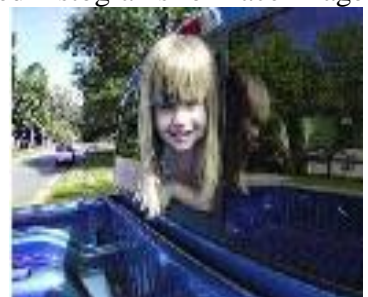

b) CLAHE image
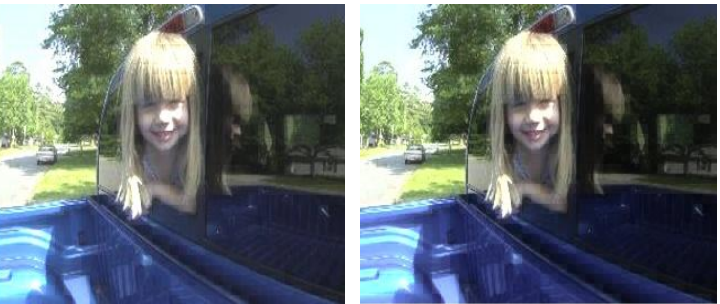

c) DCT enhanced with Y-Cb-Cr d) Enhanced with Lab Figure 8 Results of enhancement methods for lg-image16 Results of the enhancement methods for satellite lg-image16 [1] and Player image are given respectively in Figure 8, 9 and 10. It can be observed that using the CIE Lab color space enhances the image features with same histogram as $\mathrm{Y}-\mathrm{Cb}-\mathrm{Cr}$.

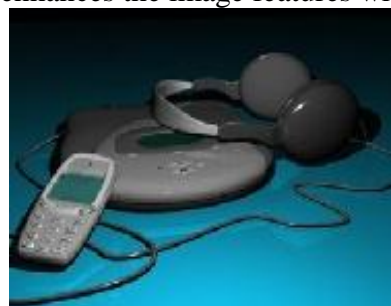

a) Original image

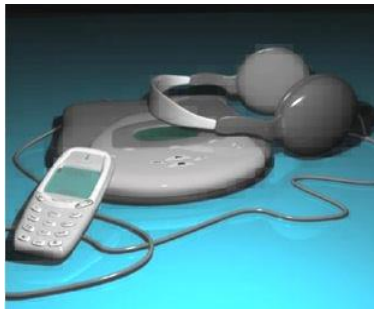

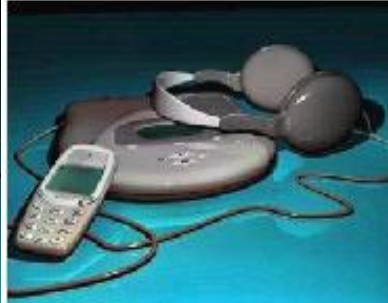

b) CLAHE image

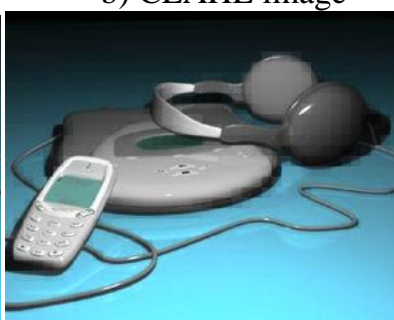

c) DCT enhanced with Y-Cb-Cr d) Enhanced with Lab Figure 9 Comparison of enhancement methods for the Player image

Table 1 and 2 gives comparison of the MSE and SNR,

$$
\begin{gathered}
M S E=\frac{1}{m n} \sum_{i=0}^{m-1} \sum_{j=0}^{n-1}[I(i, j)-K(i, j)]^{2} \\
\left.S N R=\frac{1}{m n} \sum_{i=0}^{m-1} \sum_{j=0}^{n-1}[I(i, j)) / I(i, j)-K(i, j)\right]^{2}
\end{gathered}
$$

It can be observed from the Table 1 and 2 that CLAHE method gives higher SNR and Entropy for true color image but it suffer from the color shifting and brightness change. While for images with non uniform illumination as iu player image CLAHE method gives poor performance. The standard DCT method with $\mathrm{Y}-\mathrm{Cb}-\mathrm{Cr}$ format has poor entropy and SNR. Using the LAB color space improves the performance of conventional DCT method.

Table 1 Comparison of the Signal to Noise Ratio (SNR) for enhancement methods

\begin{tabular}{|c|c|c|c|c|}
\hline S. No. & Images & $\begin{array}{c}\text { CLAHE } \\
\text { Method }\end{array}$ & $\begin{array}{c}\text { DC coefficient Scaling } \\
\text { in Y-Cb-CR space }\end{array}$ & $\begin{array}{c}\text { DC coefficient Scaling } \\
\text { L*a*b space }\end{array}$ \\
\hline 1. & Satellite & 3.5170 & 2.2329 & 2.5497 \\
\hline 2. & Faceimage1 & 5.7045 & 5.5380 & 5.5880 \\
\hline 3. & lg-image16 & 3.03895 & 2.19238 & 2.7741 \\
\hline 4. & Player & 3.44439 & 1.79331 & 1.8260 \\
\hline
\end{tabular}


Table 2 Comparison of the discrete Entropy for enhancement methods

\begin{tabular}{|c|c|c|c|c|c|}
\hline $\begin{array}{c}\text { S. } \\
\text { No. }\end{array}$ & Images & $\begin{array}{c}\text { Original } \\
\text { entropy }\end{array}$ & $\begin{array}{c}\text { CLAHE } \\
\text { method }\end{array}$ & $\begin{array}{c}\text { DC coefficient Scaling } \\
\text { in Y-Cb-CR space }\end{array}$ & $\begin{array}{c}\text { DC coefficient Scaling } \\
\text { in L*a*b space }\end{array}$ \\
\hline 1. & Satellite & 7.4684 & 7.6816 & 7.5467 & 7.6275 \\
\hline 2. & Faceimage1 & 7.7200 & 7.8518 & 7.0877 & 7.2513 \\
\hline 3. & lg-image16 & 7.2379 & 7.4395 & 7.2392 & 7.3750 \\
\hline 4. & Player & 7.3056 & 7.2477 & 7.5515 & 7.6819 \\
\hline
\end{tabular}

\section{CONCLUSION}

Paper proposed using the $\mathrm{LAB}$ color space along with the DCT based method by scaling the DC coefficients. Paper also compares the colour image enhancement methods in spatial and compressed DCT domain. The spatial domain method CLAHE suffers from the colour shifting problem although it perform significantly well for true color image in terms of entropy and SNR. The compressed domain method with DCT is free from the colour shifting. Performance of the three enhancement methods are compared based on Entropy and SNR. CLAHE method gives improved SNR for images with non uniform illumination. But for true colour images, enhancement method with LAB-DCT significantly improves the contrast and also the visual appearance of the image.

\section{REFERENCES}

[1] Jayanta M., and Sanjit K. Mitra, "Enhancement of Colour Images by Scaling the DCT Coefficients", IEEE Transactions on Image Processing, Vol. 17, No. 10, pp. 1783-1794, 2008

[2] Prateek S. Sengar, Tarun K. Rawat, Harish Parthasarathy, "Colour Image Enhancement by Scaling the Discrete Wavelet Transform.

[3] Prateek S. Sengar, Tarun K. Rawat, Harish Parthasarathy, "Colour Image Enhancement by Scaling the Discrete Wavelet Transform Coefficients", IEEE International Conference on Microelectronics, Communication and Renewable Energy (ICMiCR), 2013

[4] Irene Fondon, Mark J. J. P. van Grinsven, Clara I. Sanchez, Aurora Saez, "Perceptually Adapted Method for Optic Disc Detection on Retinal Fundus Images", Proc. of IEEE international conference pp. 279-284, CBMS 2013.

[5] Vasile V. Buzuloiu, Mihai Ciuc, Rangaraj M. Rangayan, Loic Kil, Constantin Vertan, "Histogram equalization of colour images using the adaptive neighborhood approach", Proc. SPIE 3646, Nonlinear Image Processing X, 330, 1999.

[6] K. Zuiderveld, "Contrast Limited Adaptive Histogram Equalization", Graphics Gems IV, pp. 474-485 1998

[7] P. E. Trahanias, \& A. N. Venetsanopoulos, Colour image enhancement through 3-D histogram equalization, Proc. 11th IAPR Conf. on Pattern Recognition, The Hague, Netherlands, pp. 545-548, 1992.

[8] B. A. Thomas, R. N. Strickland, \& J. J. Rodriguez, "Colour image enhancement using spatially adaptive saturation feedback", Proc. 4th IEEE Conf. on Image Processing, Santa Barbara, CA, USA, pp. 30-33, 1997

[9] Etta D, Pisano, S. Zong, R. E Jhonston “Contrast limited adaptive histogram equalization image processing to improve the detection of simulated speculation in Dense Monograms", Journal of Digital Imaging, vol. 11, No. 4, pp 193-200, 1998",

[10]. Gonzalez, RC, Woods, RE \& Eddins,2004, Digital Image processing using MATLAB, Prentice Hall.

[11] Agung W. Setiawan, Tati R. Mengko, Oerip S. Santoso, Andriyan B. Suksmono, "Colour retinal image enhancement using CLAHE", Proc. of 2013 International Conference on ICT for Smart Society (ICISS), pp. 1-3, 2013

[12] A.Saradha Devi, S. Suja Priyadharsini, S. Athinarayanan, "A Block based Scheme for Enhancing Low Luminated Images", International journal of Multimedia \& Its Applications (IJMA) Vol. 2, No. 3, pp. 49-61, 2010.

[13]. Narasimhan K, Sudarshan C R, N. Raju, “A Comparison of Contrast Enhancement Techniques in Poor Illuminated Gray Level and Colour Images", International Journal of Computer Application s0975 - 8887) Vol. 25, No .2, pp. 17-25, 2011.

[14]. K. K. Sharma, Supriya M.," Colour Image Enhancement using Nonlinear Mapping in Colour and Transform Domains", International Journal of Advanced Electronics \& Communication Systems, Is2 Vol. 12012.

[15] Michal Recky, Franz Leberl, "Windows detection using $\mathrm{K}$-means in CIE-LAB color space", IEEE International Conference on Pattern Recognition, pp. 356-359, 2010

[16] Adrian Ciobanu, Tudor Barbu "A novel Iris clustering approach using LAB color features", Proc. of IEEE $4^{\text {th }}$ International Symposium on Electrical and Electronics Engineering (ISEEE), pp. 1-4, 2013.

\section{AUTHORS}

1. Anzar Alam Have received his Bachelor's degree in Electronics and communication trend in year 2010 form MACET, Patna. He is currently pursuing M.Tech in Digital Communication, from SISTec, College Affiliated from RGPV, Bhopal India.

2. Mohd. Abdullah: Have received M. Tech degree and is currently working as Astt. Prof at SISTEC College Bhopal in Electronics and communication department

3. Ravi Shankar Mishra: Have received $\mathrm{PhD}$ degree in the VLSI field from the MANIT Bhopal, and is currently working as Head of the department ECE, SISTEC College Bhopal India 